\title{
Erratum to: Fisetin induces apoptosis in human cervical cancer HeLa cells through ERK1/2-mediated activation of caspase-8-/caspase-3-dependent pathway
}

\author{
Tsung-Ho Ying $\cdot$ Shun-Fa Yang $\cdot$ Su-Ju Tsai $\cdot$ \\ Shu-Ching Hsieh • Yi-Chang Huang • \\ Da-Tian Bau $\cdot$ Yi-Hsien Hsieh
}

Published online: 23 December 2011

(C) Springer-Verlag 2011

\section{Erratum to: Arch Toxicol}

DOI 10.1007/s00204-011-0754-6

The original publication of the article does not include the first author, Tsung-Ho Ying's additional affiliation. It is now given below for reference;

Department of Obstetrics and Gynecology, Chung Shan Medical University Hospital, Taichung, Taiwan.

The online version of the original article can be found under doi:10.1007/s00204-011-0754-6.

\section{T.-H. Ying}

Department of Obstetrics and Gynecology, School of Medicine, College of Medicine, Chung Shan Medical University,

Taichung, Taiwan

\section{T.-H. Ying}

Department of Obstetrics and Gynecology,

Chung Shan Medical University Hospital,

Taichung, Taiwan

S.-F. Yang · S.-C. Hsieh

Institute of Medicine, Chung Shan Medical University,

Taichung, Taiwan

\section{S.-F. Yang}

Department of Medical Research, Chung Shan Medical

University Hospital, Taichung, Taiwan

\section{S.-J. Tsai}

Department of Physical Medicine and Rehabilitation,

Chung Shan Medical University Hospital,

Taichung, Taiwan
S.-J. Tsai

Department of Physical Medicine and Rehabilitation, College of Medicine, Chung Shan Medical University, Taichung, Taiwan

Y.-C. Huang · Y.-H. Hsieh ( $\square)$

Institute of Biochemistry and Biotechnology,

College of Medicine, Chung Shan Medical University,

Taichung, Taiwan

e-mail: hyhsien@csmu.edu.tw

D.-T. Bau ( $\square)$

Graduate Institute of Clinical Medical Science,

China Medical University, Taichung, Taiwan

e-mail: artbau2@gmail.com

Y.-H. Hsieh

Department of Biochemistry, School of Medicine,

Chung Shan Medical University Clinical Laboratory,

Chung Shan Medical University Hospital, No. 110,

Section 1, Chien-Kuo N. Road, Taichung, Taiwan 